\title{
Factores sociales y culturales condicionan la desnutrición crónica en niños de 3 a 5 años adscritos micro red de Chupaca
}

Social and cultural factors condition chronic malnutrition in children 3 to 5 years of age

attached micro network of Chupaca

Geovanna Patricia Rojas Perales ${ }^{1}$

Layli Violeta Maravi Baldeón²

Soledad Aura Garay Quintana 3

RECIBIDO: 27 DE ABRIL DE 2020

ACEPTADO: 5 DE JUNIO DE 2020

\section{RESUMEN}

Los factores sociales y culturales son muy importantes en la vida de los seres humanos porque de ellos depende la situación de salud de las personas, sobre todo cuando se registra la desnutrición infantil como problema primordial de salud pública y

\footnotetext{
1 Maestra en Ciencias de la salud Pública y salud Pública, Trabajadora Social, Docente, Universidad Nacional del Centro del Perú, Perú; projas@uncp.edu.pe. ORCID: https://orcid.org/0000-0002-7471-3789,

Google Scholar: https://scholar.google.es/citations?view_op=new_profile\&hl=es

2 Doctora en salud Pública, Trabajadora Social, Docente, Universidad Nacional del Centro del Perú, Perú; Imaravi@uncp.edu.pe, ORCID: https://orcid.org/0000-0002-1826-1845

Google Scholar: https://scholar.google.es/citations?hl=es\&user=1G7Tt98AAAAJ

3 Doctora en Ciencias de la salud Pública y salud Pública, Trabajadora Social, Docente, Universidad Nacional del Centro del Perú, Perú; sgaray@uncp.edu.pe, ORCID: https://orcid.org/0000-0001-6219-2413

Google Scholar: https://scholar.google.es/citations?user=8QM7oWEAAAAJ\&hl=es
} 
bienestar del niño, las consecuencias son fatales en el mediano y largo plazo. El objetivo de la investigación fue: Identificar los factores sociales y culturales que condicionan la desnutrición crónica en niños de 3 a 5 años de edad adscritos a la micro red de salud Chupaca; tipo investigación básica, nivel descriptivo, diseño transaccional, la población fue de 700 madres con niños en estado de desnutrición crónica, la muestra fueron 70 madres, el instrumento fue el cuestionario. Los resultados señalan: del 40\% de las madres su nivel de escolaridad es secundaria completa, 51,42 \% viven hacinados y solo cuentan con fluido eléctrico, del 91,42 \% sus ingresos son s/.950.00 datos que son los factores sociales y como factores culturales el $80.01 \%$ de las madres tienen creencias que condicionan a la desnutrición de sus hijos, del $60 \%$ su dieta alimenticia es deficiente y el $67 \%$ de las madres muestran deficientes prácticas culturales en el cuidado del niño; en conclusión. La desnutrición de los niños es a causa de la práctica de patrones culturales alimenticios porque no son alimentados regularmente con dieta nutritiva e influencian las condiciones sociales y los recursos, así como los hábitos alimenticos y las creencias equivocadas reforzando estas conductas.

Palabras clave: Niños(as)desnutridos, factores sociales, factores culturales

\section{ABSTRACT}

Social and cultural factors are very important in the lives of human beings because the health situation of individuals depends on them, especially when child malnutrition is recorded as a primary problem of public health and child welfare, the consequences are fatal in the medium and long term. The objective of the research was: To identify the social and cultural factors that condition chronic malnutrition in children from 3 to 5 years of age enrolled in the Chupaca health micro network; basic research type, descriptive level, transactional design, the population was 700 mothers with children in a state of chronic malnutrition, the sample was 70 mothers, the instrument was the questionnaire. The results show that $40 \%$ of the mothers have completed secondary education, $51.42 \%$ live in overcrowded conditions and only have electricity, $91.42 \%$ have income above 950.00 soles, and $80.01 \%$ of the mothers have beliefs that contribute to the malnutrition of their children, $60 \%$ have a deficient 
diet and $67 \%$ of the mothers have deficient cultural practices in the care of their children. Malnutrition in children is caused by the practice of cultural eating patterns because they are not regularly fed a nutritious diet and influence social conditions and resources, as well as eating habits and mistaken beliefs, reinforcing these behaviors.

Keywords: Malnourished children, social factors, cultural factors

\section{Introducción}

Los factores sociales y culturales son condicionantes en los modos de vida de los grupos humanos determinando la situación de salud de las personas donde la desnutrición infantil se registra como problema primordial de salud pública y bienestar social del niño, con consecuencias fatales en su desarrollo integral en el mediano y largo plazo.

Gutiérrez (2011) señala que el estado nutricional es el resultado de una amplia gama de condiciones sociales y económicas y constituye un indicador muy sensible del nivel general de desarrollo. El marco conceptual de las causas de la malnutrición difundido por UNICEF plantea tres grandes grupos: a) Causas inmediatas, las relacionadas con la ingesta inadecuada de alimentos y micronutrientes y las enfermedades de tipos infeccioso; b) Causas subyacentes, relacionadas con la inseguridad alimentaria en el hogar, que tiene que ver con la poca disponibilidad de alimentos, la falta de acceso a ellos y su uso inadecuado, que a su vez, tiene que ver con patrones de inadecuada información y c) Causas básicas, relacionadas con las estructuras políticas, económicas e ideológicas en la sociedad que regulan el acceso a los recursos, la educación y las oportunidades y que finalmente determinan la pobreza de las familias, que es la raíz del problema.

La organización mundial de la salud (OMS) (2018) estima que son 180 millones de niños desnutridos en todo el mundo. Cada año la desnutrición es responsable de la muerte de niños menores de cinco años entre 3.5 y 5 millones afectando el desarrollo de un país. De ahí la importancia de abordar esta problemática social, la prevalencia de la desnutrición crónica en el país persiste, en los últimos 5 años se ha incrementado 
por ello se ha priorizado la implementación de políticas referidas a el control de la desnutrición y de la anemia, educación sobre hábitos alimenticios saludables para contribuir a mejorar la calidad de vida optima en ese sentido: el objetivo de la investigación fue Identificar factores sociales y culturales que condicionan la desnutrición crónica en niños de 3 a 5 años de edad adscritos micro red de Chupaca, la finalidad de la investigación conlleva a conocer las causas en la práctica de los estilos de vida de estas familias, la desnutrición crónica tiene efectos negativos inmediatos, como mayor probabilidad de ocurrencia de enfermedades o muerte en niños menores de cinco años, afectando el desempeño escolar, la capacidad de trabajo y ocasiona costos y pérdidas económicas a la familia.

Hipótesis del estudio: Los Factores Sociales y Culturales condicionan la desnutrición crónica en niños de 3 a 5 años adscritos a la micro red de Chupaca son; El bajo nivel de escolaridad de la madre; en la localidad aún pervive la discriminación donde el varón tiene mayor valía que la mujer, priorizando la educación de los varones, las que tienen acceso a la educación son discriminadas en el trabajo, a pesar de ello en el seno del hogar hay prácticas valiosas en bien de la familia como la colaboración y solidaridad. La diversidad de estrategias implementadas, la definición de metas educativas y la calidad del apoyo emocional parental recibido, estarían asociados con un ambiente familiar rico en capital humano y cultural (Grusec, 2002; Palacios, 1999) Citado por Muñoz Silva, Alicia (2005).

El hacinamiento; en el Perú de los 33 millones de habitantes, 9 millones viven en hacinamiento por la gran movilidad social dándose la migración generalmente del campo a la cuidad en busca de mejores condiciones de vida, creando los asentamientos humanos en las ciudades, que son cinturones de pobreza, mano de obra barata no tecnificada y para su cobijo en su mayoría rentan una habitación que es de múltiple uso y multifamiliar.

Bajos ingresos, en el país el ingreso mínimo vital es de s/. 950.00 que no cubre la canasta básica familiar haciéndose vulnerable como manifiesta la PNUD que el país es muy vulnerable, no hay empleo, mucha informalidad, relaciones de familia poco saludables, entre otros; Keynes (1983) La demanda de los individuos depende de sus ingresos, siendo la economía un elemento básico para garantizar una alimentación; hecho que lamentablemente no se cumple más aún en las zonas rurales donde 
generalmente se dedican a la agricultura, realizando la labor de peonaje donde los varones por su tarea perciben más ingreso que las mujeres, realizando la misma labor y otros oficios cuya remuneración en ocasiones esta inclusive por debajo del mínimo vital, un buen número de madres son amas de casa por lo que no perciben ningún ingreso yendo en desmedro de la familia. Rodríguez Garcés (2016) Articulo: Calidad Educativa del Ambiente Familiar y Escolaridad Materna el salario percibido de una persona es o será igual al tipo de trabajo realizado.

Creencias, En la población hay muchas practicas basadas en creencias que perjudican el desarrollo del niño o niña, consideran que los más pequeños deben comer del plato de la madre, que no es necesario las vacunas porque se enferman. Deficientes hábitos alimenticios, generalmente las madres no tienen conocimientos para balancear los alimentos, hay mucho consumo de carbohidratos, hoy en día consumen productos de escaso valor nutritivo, por la facilidad de prepararlos y comen lo que les gusta, sobre todo comida chatarra, descuidando la salud de sus hijos. La Conferencia sobre Nutrición (FAO)2 “ manifiesta que, en casi todos los países, los patrones sociales y culturales tienen una influencia notable sobre lo que consumen las personas, en el cómo preparan sus alimentos, sus prácticas alimentarias y los alimentos de su preferencia, como ejemplo es suministrar a las mujeres alimentos abundantes, densos en energía, durante los primeros meses después del parto, siendo así que algunas prácticas alimentarias tradicionales y tabúes de ciertas sociedades pueden contribuir a deficiencias nutricionales en la población. Inclusive en la primera etapa de vida de sus hijos descuidan la lactancia materna, que es de vital importancia para garantizar su adecuado desarrollo. Sanmiguel (2013) Lo más importante de la lactancia materna no es el alimento ni los factores inmunológicos, sino la relación tan especial que se establece entre el hijo y su madre, considerado de igual forma como una confirmación de un acto de amor como la emoción que siente el niño y tanto la madre, es ese vínculo afectivo y contacto físico.

Práctica de patrones culturales en el cuidado del niño, las madres no cumplen con darles a los niños la lactancia exclusiva hasta los seis meses, en ese periodo van dándoles algunos líquidos de frutas o bebidas, porque dicen que los bebés se antojan. De Garine (1997) citado por Manrique Salazar la cultura influye sobre el 
comportamiento relacionado con el consumo de alimentos y, en última instancia, sobre el estado nutricional de los individuos que integran cada población humana. La desnutrición tiene causa multifactorial de acuerdo con la dinámica que se da en cada seno familiar. González, León \& otros (2016) Estudios realizados en Latinoamérica han puesto de manifiesto que los hábitos alimentarios de los niños están determinados, fundamentalmente, por el nivel económico, educativo y la situación laboral de los padres. Esta última incide especialmente en la menor dedicación, en la preparación de las comidas y la falta de tiempo para la cocina. Además, las edades de las madres que oscilan entre los 15 a 29 años muchas de ellas se encuentran culminando la adolescencia tardía y están llenas de sentimiento juvenil (Benjamín, 1912)"Juventud que no sólo se encuentra llena de futuro, sino que siente dentro de sí la alegría y el coraje de los nuevos portadores de la cultura". La investigación desarrollada ha sido de vital importancia porque a pesar de los bastos estudios que se vienen realizando y desde diferentes perspectivas, en la actualidad la problemática de la desnutrición infantil persiste en altas tasas de incidencia y prevalencia, en este contexto.

\section{Método}

La investigación fue de tipo básico, porque selecciona y describe los factores sociales y culturales que condicionan la desnutrición de los niños(as) adscritos a la micro red salud -Chupaca, nivel descriptivo, el diseño de la investigación es no experimental y transversal.

El Método general de la investigación fue el científico porque se ha tenido en cuenta los pasos y procedimientos de la investigación, como método especifico se ha empleado el método de análisis - síntesis, lo que nos permitió analizar la información obtenida contrastando con la información de la investigación bibliográfica y realizar las consolidaciones necesarias.

La población está constituida por 700 niños(as) adscritos a la micro red de salud Chupaca, la muestra corresponde al 10\% de la población 70 madres de niños en estado de desnutrición. Los datos se recopilaron mediante la encuesta, cuyo instrumento fue el cuestionario que estuvo conformado por indicadores basados en las variables del estudio, siendo: los datos generales de las madres, los datos 
generales de los niños, los indicadores para probar los factores sociales y los fatores culturales lo que nos llevó a la demostración de la Hipótesis, para la estadística se utilizó $r$ de Pearson.

La aplicación del cuestionario fue por las investigadoras con apoyo de estudiantes que son colaboradoras en el desarrollo del estudio, la aplicación fue en equipo.

\section{Resultados}

\section{Tabal 1}

Factores sociales que condicionan la desnutrición crónica

\begin{tabular}{|c|c|c|c|}
\hline \multirow{2}{*}{$\begin{array}{l}\text { Características } \\
\text { Factores Sociales }\end{array}$} & \multicolumn{3}{|l|}{ Frecuencia } \\
\hline & Indicadores & $\mathbf{n}$ & $\%$ \\
\hline Grupo & Adolescente (15-19) & 5 & 7,14 \\
\hline \multirow[t]{4}{*}{ etario } & Joven (20-24) & 29 & 41,42 \\
\hline & Adulto joven (25 -29) & 28 & 40.00 \\
\hline & Adulto medio $(30 \ldots)$ & 5 & 7,14 \\
\hline & Adulto mayor (60 a más) & 3 & 5,71 \\
\hline Nivel de & Sin escolaridad & 0 & 0 \\
\hline \multirow[t]{6}{*}{ escolaridad } & Primaria incompleta & 25 & 35.71 \\
\hline & Primaria completa & 8 & 11,42 \\
\hline & Secundaria Incompleta & 5 & 7,14 \\
\hline & Secundaria Completa & 28 & 40.0 \\
\hline & Superior Incompleto & 4 & 5,71 \\
\hline & Superior Completo & 0 & 0 \\
\hline \multirow[t]{3}{*}{$\begin{array}{l}\text { Vivienda } \\
\text { segura }\end{array}$} & $\begin{array}{l}\text { Agua, desagüe, fluido, ambientes básicos para c } \\
\text { función }\end{array}$ & 7 & 10,00 \\
\hline & $\begin{array}{l}\text { Agua, fluido eléctrico ambientes limitados para c } \\
\text { función }\end{array}$ & 27 & 38,57 \\
\hline & Fluido eléctrico, una sola habitación compartida & 36 & 51,42 \\
\hline \multirow[t]{2}{*}{ Ingresos } & $s / .950 .00$ & 64 & 91,42 \\
\hline & $s / .950 .00$ a s/.1350 & 5 & 7,14 \\
\hline
\end{tabular}




\begin{tabular}{llll}
\hline & s/.1350 a s/. 1650. & 1 & 1,42 \\
& s/. 1650 a mas & 0 & 0 \\
\hline Ocupación & Ama de casa & 56 & 80,01 \\
& Comerciante & 11 & 15,71 \\
& Agricultor (ra) & 3 & 4,28 \\
\hline Carga & 1-3 miembros de familia & 24 & 34,28 \\
familiar & 3-5 miembros de familia & 29 & 41,42 \\
& 6- 8 miembros de familia & 17 & 24,28 \\
& Más de 8 miembros de familia & 24 & 34,28 \\
\hline
\end{tabular}

Las edades del grupo etario (Jóvenes) las madres oscilan entre (20 a 24 años) representada el $41,42 \%$, seguido por el $40 \%$ del grupo de madres adulto jóvenes (25 a 29 años) haciendo un total de $81.42 \%$ y siendo en un minino de adultas medio y mayor que es el 12.85\% (30 a 60 o más años); el 40\% ha cursado su nivel de escolaridad secundaria completa, el $91,42 \%$ sus ingresos económicos son de s/. 950.00 perciben bajos ingresos económicos, del 80,01\% su ocupación es de ama de casa, se dedican a la atención de sus hijos y del hogar, sin embargo, sus menores hijos tienen desnutrición crónica, del $41,42 \%$, su carga familiar es de 3 a 5 miembros por familia, a su vez cada una de estas tienen hijos menores de 5 años con desnutrición crónica.

\section{Tabla 2}

Factores culturales que condicionan la desnutrición Crónica en niños de 3 a 5 años

\section{Características}

\section{Frecuencia}

\begin{tabular}{|c|c|c|c|c|}
\hline \multirow[t]{2}{*}{ Indicadores } & \multicolumn{2}{|c|}{$\begin{array}{l}\text { De } \\
\text { acuerdo }\end{array}$} & \multicolumn{2}{|c|}{$\begin{array}{l}\text { En } \\
\text { desacuerdo }\end{array}$} \\
\hline & $\mathbf{n}$ & $\%$ & $\mathbf{n}$ & $\%$ \\
\hline $\begin{array}{l}\text { La niña y los niños } \\
\text { consumen diferente } \\
\text { cantidad de alimentos, } \\
\text { los varones consumen en } \\
\text { mayor cantidad por ser }\end{array}$ & 56 & 80 & 14 & 20 \\
\hline
\end{tabular}


varones.

\section{Creencias \\ del consumo de alimentos}

No es bueno darles

siempre alimentos nutritivos, le pl hacerles daño por

exceso.

No comen alimentos

nutritivos, consumen en

pocas cantidades, es mejor

darles comidas que les gusta.

Los vegetales, las carnes,

leche,

huevos

previenen
84,2

$11 \quad 15,71 \quad 59 \quad 84,2$

desnutrición

Cuando el niño se enferma se le d $15 \quad 21,43 \quad 55 \quad 78,5$

comidas que le gustan

$\begin{array}{lllll}\text { De vez en cuando hay que } & 55 & 78,5 & 15 & 21,4\end{array}$

darle un golpecito para que con

no ser tolerante,

hablar fuerte

Dieta

Los niños(as)comen sus

70

100

alimentos más de 3 veces

al día

balanceados

$10 \quad 14,28 \quad 60$

85,7

(carme, menestras y

frutas) durante la semana

escolar.

Frecuentemente consumen carne $\begin{array}{lllll}23 & 32,86 & 47 & 67,1\end{array}$

Yogur, leche, queso

huevo verduras en su

dieta alimenticia

$\begin{array}{lllll}\text { Frecuentemente (4 veces } & 48 & 68,57 & 22 & 31,4\end{array}$

a la semana) comen frutas

$\begin{array}{lllll}\text { Lactancia materna hasta } & 8 & 11,42 & 56 & 80\end{array}$

Prácticas

culturales en

el cuidado

del niño

\begin{tabular}{lllll} 
los 6 meses mínimos & & & & \\
\hline Afiliación a un seguro & 31 & 44,28 & 39 & 4,2 \\
\hline Control de salud periódico & 29 & 41,42 & 41 & 58,5 \\
\hline Alimentación balanceada & 26 & 37,14 & 44 & 15,7 \\
\hline $\begin{array}{l}\text { Interés de la madre en informació } \\
\text { nutrición }\end{array}$ & 55,71 & 31 & 44,2 \\
(radio, la tv y centro de & & & \\
salud) & & & & \\
\end{tabular}


El $80.01 \%$ de las madres tienen creencias sobre el consumo de alimentos que condicionan a la desnutrición de sus hijos; mayor al 70\% la dieta alimenticia de los niños no incluye proteínas, ni vitaminas y el $80,1 \%$ de las madres muestran que no le dieron lactancia materna hasta los 6 meses, el 58,57\% se descuidan del control de la salud de su niño, el 78,57\% de las madres consideran que es necesario dar gritos, castigarlos para educarlos son actitudes que van en contra del bienestar del niño, estos factores en global contribuyen a la prevalencia de la desnutrición crónica. Las versiones con que justifican "...No siempre se le trata con cariño y tolerancia al niño porque si no se malcría.... siempre es necesario ...un toquecito para que aprenda sino no hacen caso, hablarles un poco fuerte......" el 100\% refieren que los niños menores de 5 años en estado de desnutrición consumen más de 3 veces sus alimentos al día.

\section{Discusión}

H 1. Los Factores sociales condicionan la desnutrición crónica en niños de 3 a 5 años adscritos micro red de Chupaca son; el bajo nivel de escolaridad, edad de la madre, ocupación, hacinamiento y bajos ingresos económicos.

La edad de la madre de los niños(as) con desnutrición crónica está entre los 20 a 24 años de edad, igual al 41,42\%, y el 40\% entre 25 a 29 años de edad, quiere decir que son madres jóvenes haciendo un total al $81,42 \%$ muchas de ellas se encuentran culminando la adolescencia tardía, afianzando sus relaciones sociales en torno al contexto histórico social en el que viven y en muchos casos determina sus comportamientos, de acuerdo a la teoría de la juventud (Benjamín, 1912)"Juventud que no sólo se encuentra llena de futuro, sino que siente dentro de sí la alegría y el coraje de los nuevos portadores de la cultura... Este sentimiento juvenil se convierte en una forma de pensar compartida por todos, en una brújula de la vida" si en esta categoría etaria existe una proyección de vida en el caso de más madres con hijos desnutridos esa brújula se centra en la crianza de su hijo, no lo hacen acorde al rigor de las funciones paternales por varios factores de visión de la vida, sus actitudes se centran en aspectos distractores como la televisión, conversando o cumpliendo con los quehaceres de la casa, limpiando, cocinado ,ordenando, trabajando; así mismo 
esto está relacionado a las edades de las madres jóvenes en un contexto marcado por las incertidumbres y las paradojas de la sociedad postmoderna; del 91,42\% sus ingresos económicos son bajos para solventar la canasta básica familiar (s/. 950.00) Keynes (1983) "La demanda de los individuos depende de sus ingresos". El ingreso es el producto del gasto, el ingreso depende de la ocupación laboral y tiempo que se le dedica para la generación de estos, asociado a escalas por oficios, empleos y tipos de contratos. Si aumenta la actividad, aumentan los ingresos y la demanda. En estas condiciones las madres se dedican a la labor de ama de casa en un $80,01 \%$ sin embargo está condicionado a que sus menores hijos tengan desnutrición crónica el $41,42 \%$ tiene una carga familiar de 3 a 5 miembros y sus ingresos son deficientes.

Gutiérrez, Wilfredo (2011) señala que..." El estado nutricional es el resultado de una amplia gama de condiciones sociales y económicas y constituye un indicador muy sensible del nivel general de desarrollo. El marco conceptual de las causas de la malnutrición difundido por UNICEF plantea tres grandes grupos: a) Causas inmediatas, las relacionadas con la ingesta inadecuada de alimentos y micronutrientes y las enfermedades de tipos infeccioso; b) Causas subyacentes, relacionadas con la inseguridad alimentaria en el hogar, que tiene que ver con la poca disponibilidad de alimentos, la falta de acceso a ellos y su uso inadecuado, que a su vez, tiene ver con patrones de inadecuada información y c) Causas básicas, relacionadas con las estructuras políticas, económicas e ideológicas en la sociedad que regulan el acceso a los recursos, la educación y las oportunidades y que finalmente determinan la pobreza de las familias, que es la raíz del problema".

Los padres cumplen un rol fundamental y difícilmente sustituible en el seno de la familia, en la crianza de sus hijos, no solo deben proporcionar cuidado y protección, sino tienen que cumplir con la función de educadores cuando el ambiente familiar es precario está en directa relación con el perfil de idoneidad y competencia de los padres, siendo el nivel de escolaridad alcanzado, sobre todo por las madres, un importante predictor de la modalidad y calidad de los estilos educativos que se realicen dentro del hogar. La diversidad de estrategias implementadas, la definición de metas educativas y la calidad del apoyo emocional parental recibido, estarían asociados con un ambiente familiar rico en capital humano y cultural (Grusec, 2002; 
Palacios, 1999). Los padres con un bajo nivel socioeconómico y reducido capital cultural evidenciarán dificultades para proveer un ambiente de calidad, estimulante y eficiente en la instalación de habilidades y actitudes, precisando, en mayor medida, del apoyo externo que posibilite el reforzamiento e implementación de estrategias educativas más efectivas. El adelantamiento en el proceso de escolarización a edades cada vez más tempranas es expresión de este fenómeno, donde, mediante la acción sistemática, formal e intencionada, se busca compensar las deficiencias del entorno socioeducativo del niño. Rodríguez Garcés (2016) Articulo: Calidad Educativa del Ambiente Familiar y Escolaridad Materna el salario percibido de una persona es o será igual al tipo de trabajo realizado. De acuerdo al estudio de investigación corroboramos que los ingresos son mínimos para sostener una canasta básica familiar por las madres de los niños con estado de desnutrición quienes se dedican al cuidado de sus hijos siendo su rol de ama de casa, los ingresos económicos depende de la pareja que le asigna para el gasto de canasta básica familiar como lo ingresos son bajos estos no les permite proveer recursos alimenticos con calidad, con nutrientes requeridos, los recursos económicos permiten adquirir productos alimenticos con bajos nutrientes y estos recursos están en función a los ingresos y la distribución de los mismos priorizando la alimentación, en ese sentido podemos sostener que los factores sociales identificados como el bajo nivel de escolaridad de la madre, los escasos ingresos económicos, el hacinamiento en el que viven los niños de 3 a 5 años con desnutrición crónica son factores que siguen siendo determinantes que condicionan su estado de desarrollo integral porque padecen de desnutrición crónica por lo tanto se demostró la hipótesis especifica 1.

H2. Los Factores culturales que condicionan la desnutrición crónica en niños de 3 a 5 años adscritos micro red de Chupaca es; creencias en el consumo de alimentos deficiente dieta alimenticia, patrones culturales en el cuidado del niño.

El $80.01 \%$ de las madres tienen creencias sobre el consumo de alimentos que afectan a la desnutrición de sus hijos, el 70\% de ellas manifiesta, la dieta alimenticia de los niños no incluye proteínas, ni vitaminas siendo deficiente, el $50 \%$ de las encuestadas consumen alimentos con bajo valor nutritivo, no siendo lo suficiente en 
la dieta alimenticia, por ello presentan estado de desnutrición o simplemente no están balanceados correctamente para causar efectos positivos porque las madres encargadas de la preparación de los alimentos no lo realizan adecuadamente acorde con la técnica y procedimientos desde el enfoque de salud y arte sino lo realizan a su manera o forma de ser sentir empoderadas en sus cimientos y creencias, normas, valores, arte y conocimientos que devienen desde sus familias paternas, las prácticas alimenticias están fundamentadas en creencias equivocadas sobre la alimentación, también responde a la cultura alimenticia dado por el consumismo del medio y del entorno, hoy en día se consumen productos de escaso valores nutritivos, la facilidad de prepararlos y comen algo que les gusta y el $95 \%$ de las encuestadas saben que no deben darles, que deben tener cuidados especiales cuando los niños se enferman, pero aun así en un 4,28\% no toman conciencia de estos actos y no cuidan la salud nutricional de los menores a su cargo. Sustentamos con la Conferencia sobre Nutrición (FAO)2 “ manifiesta que, en casi todos los países, los patrones sociales y culturales tienen una influencia notable sobre lo que consumen las personas, en el cómo preparan sus alimentos, sus prácticas alimentarias y los alimentos de su preferencia, como ejemplo es suministrar a las mujeres alimentos abundantes, densos en energía, durante los primeros meses después del parto, siendo así que algunas prácticas alimentarias tradicionales y tabúes de ciertas sociedades pueden contribuir a deficiencias nutricionales en la población. " estas prácticas repercuten justamente en las prácticas alimenticias que no proporcionan contenidos proteicos en la dieta alimenticia que ingesta el niño más aún si come alimentos menos de 3 veces al día lo cual es perjudicial en la salud de este y su posterior desarrollo, por lo tanto, las madres de estos niños que son las encargadas de brindar la alimentación esencial como es la lactancia materna no les han brindado esta alimentación básica de manera adecuada, evidenciándose que estos niños no han recibido los 6 meses como mínimo como dice la teoría. Sanmiguel (2013) Lo más importante de la lactancia materna no es el alimento ni los factores inmunológicos, sino la relación tan especial que se establece entre el hijo y su madre, considerado de igual forma como una confirmación de un acto de amor como la emoción que siente el niño y tanto la madre, ese vínculo afectivo y contacto físico. En este caso son pocos los niños que consumen alimentos nutritivos en su dieta 
alimenticia o las cantidades proteicas no son lo suficiente para el estado de desnutrición en la que se encuentran o los procedimientos de preparación no son los adecuados, la madre al preparar los alimentos tiene sus propias preferencias, rechazo a algunos alimentos, otras son conservadoras de sus tradiciones y otras innovadoras en la preparación de los alimentos. El consumo de los alimentos se origina por los cambios sociales y económicos, pero es muy difícil cambiar los hábitos alimenticos cuando ya está establecido como parte de los patrones culturales, De Garine (1997) citado en Manrique, E. (2005) la cultura influye sobre el comportamiento relacionado con el consumo de alimentos y, en última instancia, sobre el estado nutricional de los individuos que integran cada población humana y el $80,1 \%$ de las madres muestran que no le dieron lactancia materna hasta los 6 meses, el $58,57 \%$ se descuidan del control de la salud que ejercen en su niño, el $78,57 \%$ de las madres consideran que de vez en cuando a los niños se les debe dar gritos, castigos para educarlos, son actitudes que van en contra del bienestar del niño La crianza de los hijos durante la primera infancia es de vital importancia para lo cual el rol que cumplen las madres es primordial especialmente con acciones que contribuyan al desarrollo integral del niño como la lactancia materna, acceso a un seguro del niño y el trato afectuoso al niño en ese sentido el estudio realizado no ha evidenciado datos favorables porque las respuestas están en correspondencia a los estilos de vida de las familias especialmente de las madres de los niños con estado de desnutrición crónica corroboramos esta información con el enfoque Desarrollo integral del niño OPS (2015) Los niños de la Región de las Américas son su principal recurso y el reconocimiento y la protección de sus necesidades específicas y derechos humanos son esenciales para un desarrollo efectivo y las madres no toman importancia de este escenario del niño esto responde a su forma de percibir la realidad también aporta desde la salud pública (con el modelo ecológico de la salud) se han incorporado determinantes que influyen en la salud de las personas, que se entiende ahora como la consecuencia de múltiples conductas y contextos culturales y sociales la niñez representa hoy un período de gran vulnerabilidad en el cual el organismo es especialmente sensible a factores favorables y desfavorables del entorno y con un comportamiento variable ante los determinantes de la salud, en ese sentido se demostró la hipótesis especifica 2. 
HG. Los Factores sociales y culturales que condicionan la desnutrición crónica en niños de 3 a 5 años adscritos micro red de Chupaca son el bajo nivel de escolaridad de la madre, el hacinamiento, bajos ingresos y creencias sobre el consumo de alimentos deficientes alimentación balanceada y creencias culturales en el cuidado del menor.

La influencia de la cultura es de gran relevancia en la vida de las personas, incluyendo tanto sus creencias y percepciones, como sus valores, emociones, en este caso en particular, sobre la decisión que puedan tomar para el cuidado de sus hijos a fin de brindar una nutrición adecuada, en cantidad y en calidad, es clave para el buen desarrollo físico e intelectual del niño, un niño que sufre desnutrición ve afectada su supervivencia, el buen funcionamiento y desarrollo de su cuerpo y de sus capacidades cognitivas e intelectuales. La desnutrición es un concepto diferente de la malnutrición, que incluye tanto la falta como el exceso de alimentos.

\section{Conclusiones}

Los factores sociales participan de manera trascendental en la crianza de los niños con estado de desnutrición crónica siendo las madres quienes presentan bajo nivel de escolaridad que oscilan entre primaria incompleta y secundaria completa, los bajos ingresos mensuales que obtienen, que no cubre la canasta básica familiar y las condiciones de hacinamiento familiar no favorecen su condición de salud del niño con desnutrición.

Los factores culturales están condicionados por el perfil de la madre que interviene de manera importante en la alimentación del niño y por su perfil cultural, en la práctica alimenticia repercute negativamente en la salud del niño por los deficientes conocimientos y creencias de sobre la alimentación y nutrición de su hijo.

Las diversas circunstancias sociales y culturales se traducen en factores que influyen en la dieta alimenticias que condicionan la desnutrición crónica en niños menores de 5 años, los niños consumen alimentos y recibe atención deficiente de la madre por la práctica de patrones culturales de los hábitos alimenticios, los ingresos bajos 
dándose en familias jóvenes, teniendo como efecto el estado de desnutrición en el que se encuentran sus hijos.

\section{Referencias}

Benjamin, W. (1912) teoría de la Juventud. Citado por Feixa,C.(2006) Recuperado de http://www.scielo.org.co/scielo.php?script=sci_arttext\&pid=S1692$715 \times 2006000200002$

Carles Feixa (2014) Teorías de la Juventud contemporánea. Revista Latinoamericana de Ciencias Sociales. Recuperado de http://www.scielo.org.co/scielo.php?script=sci_arttext\&pid=S1692$715 \times 2006000200002$

De Garine y Vargas, (1997) citado por Manrique, E. y Salazar, J. (2005)Dimensión Social De La Desnutrición Apuntes Para Discusión Cultura Científica JDC Recuperado de http://www.revistasjdc.com/main/index.php/ccient/article/view/174/167

Fernández, D. y Montenegro G. (2014) Factores Socioculturales del cuidador familiar relacionados con el estado nutricional del niño de 1 a 5 años en el sector Jaén, Perú. (Tesis de pregrado). Universidad Nacional de Cajamarca.

Grusec, W. (2002); Palacios, J. (1999). Citado por Muñoz Silva, Alicia. La familia Como Contexto de Desarrollo Infantil. Dimensiones de Análisis para la Intervención Educativa y Social. Portularia vol.V, n²-2005,[147-163], issn15780236. (c) Universidad de Huelva. Recuperado de https://educrea.cl/wpcontent/uploads/2017/01/DOC2-familia.pdf

Gutiérrez, W. (2011). La desnutrición en la niñez en el Perú: factores condicionantes y tendencias. Rev. Perú. med. exp. salud publica v.28 n.2 Lima abr./jun. 2011 Recuperado de https://www.unicef.es/sites/unicef.es/files/Dossierdesnutricion.pdf INEl: Encuesta Demográfica y de Salud Familiar 2016 (ENDES 2016) Araos, C., Correa, V. (2004) La escuela hace la diferencia.

Jiménez-Benítez (2010) Análisis de determinantes sociales de la desnutrición en Latinoamérica versión On-line ISSN 1699-5198versión impresa ISSN 0212-1611. 
Nutr. Hosp. vol.25 supl.3 Madrid oct. Recuperado de

http://scielo.isciii.es/scielo.php?script=sci_arttext\&pid=S0212-

16112010000900003

Keynes (1983) teoría de la ocupación, interés y dinero. Recuperado de

http://biblio.econ.uba.ar/opactmpl/bootstrap/Textocompleto/Teor\%C3\%AD a\%20general\%20

Martínez, Fernández, (2007) Impacto social y económico de la desnutrición infantil en Centroamérica y República Dominicana. Recuperado por https://www.google.com/search?q=Impacto+social+y+econ\%C3\%

Muñoz Silva, Alicia. La familia Como Contexto de Desarrollo Infantil. Dimensiones de Análisis para la Intervención Educativa y Social. Portularia vol.V, n²-2005, [147163], issn 1578-0236. (C) Universidad de Huelva https://educrea.cl/wpcontent/uploads/2017/01/DOC2-familia.pdf

Plan Nacional para la reducción de la desnutrición crónica infantil y la prevención de la anemia en el país 2014-2016. Recuperado de http://www.un.org/es/events/childrenday/pdf/derechos.pdf Consultada el 15 julio 2017

Rodríguez Garcés (2016) Articulo Calidad Educativa del Ambiente Familiar y Escolaridad Materna Centro de Investigación y Desarrollo en Computación e Informática Educativa (CIDCIE) Universidad del Bío-Bío.

Sanmiguel (2013) Modelo teórico con enfoque de enfermería cultural sobre lactancia materna Recuperado de http://ve.scielo.org/scielo.php?script=sci_arttext\&pid=\$131671382013000100007

SESAN (Secretaría de Seguridad Alimentaria y Nutricional), UNICEF (Fondo de las Naciones Unidas para la Infancia) (2014) Factores socioculturales que inciden en la desnutrición crónica. Guatemala.

UNICEF. "La desnutrición infantil condena a un país a la eterna pobreza". Artículo. New York, 2006. [en línea] Perú. 2010. [Consultado el 15 de agosto del 2013] URL. Recuperado de http/ /www. bvsde. ops-oms. org/texcorn/nutrición/ di pticoll. pdf 


\section{8}

Vallejo-Solarte. (2016) Estado nutricional y determinantes sociales en niños entre 0 y 5 años de la comunidad de Yunguillo y de Red Unidos, Mocoa, Colombia. Rev Uni. salud.;18(1):113-125. Recuperado de http://www.scielo.org.co/pdf/reus/v18nl/v18nlal2.pdf 